\title{
A Sparse Modulation Signal Bispectrum Analysis Method for Rolling Element Bearing Diagnosis
}

\author{
Guangbin Wang $\mathbb{D}^{1},{ }^{1}$ Fengshou Gu $\left(\mathbb{D}^{2},{ }^{2}\right.$ Ibraham Rehab, ${ }^{2}$ Andrew Ball, ${ }^{2}$ and Long Li ${ }^{1}$ \\ ${ }^{1}$ Health Maintenance for Mechanical Equipment Key Laboratory of Hunan Province, Hunan University of Science and Technology, \\ Xiangtan 411201, China \\ ${ }^{2}$ Center for Efficiency and Performance Engineering, University of Huddersfield, Queensgate, Huddersfield HD1 3HD, UK
}

Correspondence should be addressed to Guangbin Wang; jxxwgb@126.com

Received 7 July 2017; Accepted 21 February 2018; Published 19 April 2018

Academic Editor: Jussi Sopanen

Copyright (c) 2018 Guangbin Wang et al. This is an open access article distributed under the Creative Commons Attribution License, which permits unrestricted use, distribution, and reproduction in any medium, provided the original work is properly cited.

\begin{abstract}
Modulation signal bispectrum (MSB) analysis is an effective method to obtain the fault frequency for rolling bearing, but harmonics make fault frequency dense and even frequency aliasing. Carrier frequency of bearing is generally determined by its structure and inherent characteristics and changes with the increase of the damage degree, so it is hard to be accurately found. To solve these problems, this paper proposes a sparse modulation signal bispectrum analysis method. Firstly the vibration signal is demodulated by MSB analysis and its bispectrum is obtained. After the frequency domain filtering, the carrier frequency is computed based on the characteristics of energy concentration at the carrier frequency on MSB. By shift-frequency MSB (SF-MSB), the carrier frequency is moved to the coordinate origin, the entire MSB is shifted for the same distance, and SF-MSB is obtained. At last, the bispectrum is shifted to the frequency zero point and diagonal slices are performed to obtain a sparse representation of MSB. Experimental results show that sparse MSB (S-MSB) method can not only eliminate the interference of harmonic frequency, but also make the extracted characteristic frequency of fault more obvious.
\end{abstract}

\section{Introduction}

Bearings are the important parts and easily damaged parts of rotating machine and have received massive attention. Because rolling bearing fault vibration signal is generally weak, nonstationary, nonlinear signal, how to extract fault information is the key to rolling bearing fault diagnosis.

Bispectrum analysis is a high-order spectrum analysis method, which can effectively solve the phase blind problem of power spectrum analysis as second-order statistics; its biggest advantage is that it can preserve the phase characteristic information of the analyzed signal when detecting, estimating, and reconstructing a signal in a low SNR environment, so it is a powerful tool for dealing with nonlinear and non-Gaussian signals [1].

However, bispectrum analysis generally requires the signal to be steady-state; for unsteady or cyclostationary signals, the analysis results are not accurate enough. So many scholars had proposed some improved algorithms based on the bispectrum analysis for different research objects and specific questions, like wavelet domain bispectrum analysis [2-4], order bispectrum analysis [5], vector bispectrum analysis [6], cyclic bispectrum analysis $[7,8]$, and so on [9-11]. In 2004, a new AM detector and its normalized form are proposed and defined [12]; Gu et al. named this method as the modulation signal bispectrum (MSB) analysis and achieved fault diagnosis of downstream mechanical equipment using electrical motor current signal based on MSB in 2011 [13]. After that, their team continued to deeply study, analyze, and improve on the MSB method and also achieved a series of results [14-18].

For the fault diagnosis of the rolling bearing, MSB analysis is effective in obtaining the fault frequency, but when fault signal contains multiple harmonics, fault frequency and interference signal frequency of the bearing exist simultaneously, which may make fault frequency dense and even frequency aliasing or distorting. Carrier frequency of bearing is generally determined by its structure and inherent characteristics and changes with the increase of the damage degree, so it is hard to be accurately found. To solve 
these problems, this paper proposes a sparse MSB analysis method, which finds computing method of carrier frequency and sparse demodulation method on the rolling bearing. Simulation and experiments show that sparse MSB method can extract fault characteristic frequency effectively.

\section{Shift-Frequency Modulation Signal Bispectrum (SF-MSB)}

2.1. Conventional Bispectrum (CB). Suppose $x(t)$ is a zeromean stationary signal; the second-order cumulant $C_{2 x}(\tau)$ and third-order cumulant $C_{3 x}(\tau)$ can be calculated as

$$
\begin{aligned}
& C_{2 x}(\tau)=E\left\langle x^{*}(t) x(t+\tau)\right\rangle \\
& C_{3 x}(\tau)=E\left\langle x^{*}(t) x\left(t+\tau_{1}\right) x\left(t+\tau_{2}\right)\right\rangle,
\end{aligned}
$$

where $E\langle\rangle$ is the expectation operator, * denotes complex conjugate, and $\tau$ is time delay. Using one-dimensional and two-dimensional Fourier transforms of cumulant $C_{2 x}(\tau)$ and cumulant $C_{3 x}(\tau)$, the power spectrum and the bispectrum of $x(t)$ can be obtained as shown below:

(a) Power spectrum

$$
S(\omega)=E\left\langle X(\omega) X^{*}(\omega)\right\rangle .
$$

(b) Bispectrum

$$
B\left(\omega_{1}, \omega_{2}\right)=E\left\langle X\left(\omega_{1}\right) X\left(\omega_{2}\right) X^{*}\left(\omega_{1}+\omega_{2}\right)\right\rangle .
$$

In (3), $\omega_{1}, \omega_{2}, \omega_{1}+\omega_{2}$ indicates three individual frequency components achieved from Fourier series integral. From (2) and (3), it can be seen that bispectrum of $x(t)$ contains both magnitude and phase information of signal, but power spectrum contains only energy information. So bispectrum can suppress random noise significantly if the frequency components at $\omega_{1}, \omega_{2}, \omega_{1}+\omega_{2}$ are independent components, but when these frequency components are nonlinearly coupled to each other, this nonlinear coupling is indicated by a peak in the bispectrum at the bifrequency $B\left(\omega_{1}, \omega_{2}\right)$, and the statistical averaging will not lead to a zero value in the bispectrum [13].

2.2. Modulation Signal Bispectrum (MSB). MSB has the capability to enhance nonlinear components and suppress random noise by detecting phase coupling in modulation signal. The definition of MSB can be described by

$$
\begin{aligned}
& B_{\mathrm{MS}}\left(\omega_{1}, \omega_{2}\right) \\
& \quad=E\left\langle X\left(\omega_{1}+\omega_{2}\right) X\left(\omega_{1}-\omega_{2}\right) X^{*}\left(\omega_{2}\right) X^{*}\left(\omega_{2}\right)\right\rangle .
\end{aligned}
$$

The magnitude and phase of MSB can be expressed as

$$
\begin{aligned}
& A_{\mathrm{MS}}\left(\omega_{1}, \omega_{2}\right) \\
& =E\left\langle\left|X\left(\omega_{1}+\omega_{2}\right)\right|\left|X\left(\omega_{1}-\omega_{2}\right)\right|\left|X^{*}\left(\omega_{2}\right)\right|\left|X^{*}\left(\omega_{2}\right)\right|\right\rangle \\
& \varphi_{\mathrm{MS}}\left(\omega_{1}, \omega_{2}\right) \\
& =\varphi\left(\omega_{1}+\omega_{2}\right)+\varphi\left(\omega_{1}-\omega_{2}\right)-\left|\varphi\left(\omega_{2}\right)\right|-\left|\varphi\left(\omega_{2}\right)\right| .
\end{aligned}
$$

Signals have quadratic phase coupling characteristics; that is, their phases are related as follows:

$$
\begin{aligned}
& \varphi\left(\omega_{1}+\omega_{2}\right)=\varphi\left(\omega_{1}\right)+\varphi\left(\omega_{2}\right) \\
& \varphi\left(\omega_{1}-\omega_{2}\right)=\varphi\left(\omega_{1}\right)-\varphi\left(\omega_{2}\right) .
\end{aligned}
$$

The total phase of MSB will be zero and MSB amplitude will be the product of the four magnitudes, which is the maximum of the complex product. Therefore, a bispectral peak will appear at $\left(\omega_{1}, \omega_{2}\right)$ [13]. If these components are not coupled but they have random distribution, the magnitude of MSB will be close to zero. So wideband noise in bearing vibration signals needs to be suppressed effectively so that the discrete frequency components can be obtained more accurately [18].

2.3. Comparison between $C B$ and MSB for Analyzing Modulation Signal. The modulation signal $x(t)$ may be defined as follows:

$$
\begin{aligned}
& x(t)=a(t) \cos \left(\omega_{c} t+\theta\right) \\
& a(t)=A_{0}+A_{1} \cos \left(\omega_{m} t+\varphi\right) .
\end{aligned}
$$

In (7), $\omega_{c}$ is the carrier frequency and $\omega_{m}$ is the modulation frequency, $A_{0}$ and $A_{1}$ are arbitrary constant amplitudes, and $\theta$ and $\varphi$ are arbitrary constant phases. Fourier transform of $x(t)$ is

$$
\begin{aligned}
X(\omega)= & A_{0} \delta\left(\omega-\omega_{c}\right) e^{j \theta}+\frac{A_{1}}{2} \delta\left(\omega-\omega_{c}-\omega_{m}\right) e^{j(\theta+\varphi)} \\
& +\frac{A_{1}}{2} \delta\left(\omega-\omega_{c}+\omega_{m}\right) e^{j(\theta-\varphi)}
\end{aligned}
$$

Theoretically, from (3) and (8), nonzero values can be only obtained at the intersection points of the three group lines shown in (9) and in Figure 1(a), and other points except for these intersection points are zero on the whole $\omega_{1}-\omega_{2}$ plane [11]. Similarly, from (4) and (8), the MSB's intersection points may be obtained, described with the red points as shown in Figure 1(b)

$$
\begin{array}{r}
\omega_{1}=\omega_{c}, \omega_{c}+\omega_{m}, \omega_{c}-\omega_{m} \\
\omega_{2}=\omega_{c}, \omega_{c}+\omega_{m}, \omega_{c}-\omega_{m} \\
\omega_{1}+\omega_{2}=\omega_{c}, \omega_{c}+\omega_{m}, \omega_{c}-\omega_{m} .
\end{array}
$$

Figures 1(a) and 1(b) are the theoretical result of $\mathrm{CB}$ and MSB analysis, respectively; 5 nonzero intersection points in Figure 1(b) are equivalent to discarding redundant 12 nonzero points out of circles A, B, and C in Figure 1(a) firstly; then the intersection points in circle $\mathrm{A}$ and circle $\mathrm{B}$ are moved to circle $\mathrm{C}$. The intersection points' coordinates of the MSB are $\left(0, \omega_{c}-\omega_{m}\right),\left(0, \omega_{c}\right),\left(0, \omega_{c}+\omega_{m}\right),\left(\omega_{c}, \omega_{m}\right)$, and $\left(\omega_{c},-\omega_{m}\right)$.

2.4. Shift-Frequency MSB (SF-MSB). If the idea of this moving frequency persists, we can move the 5 intersection points in Figure 1(b) $\omega_{c}$ away to zero point along the $\omega_{1}$-axis 


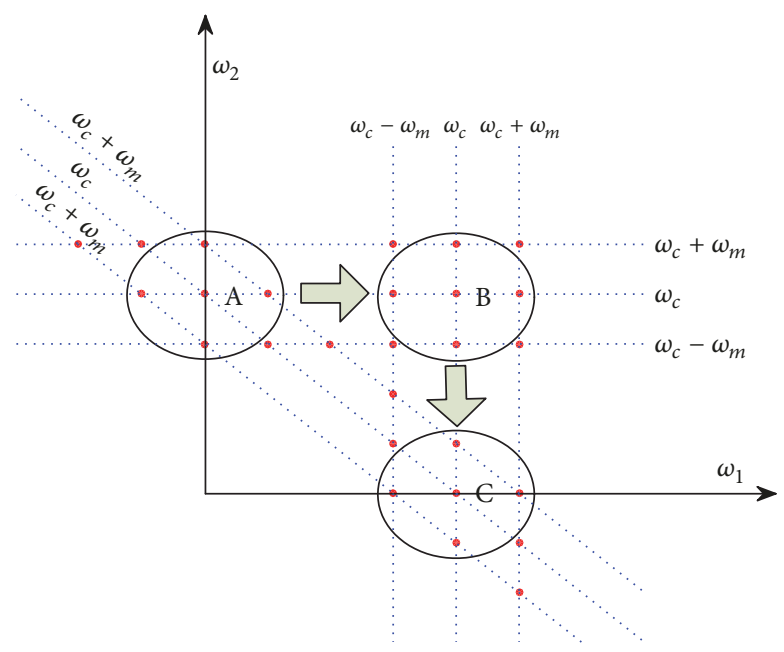

(a)

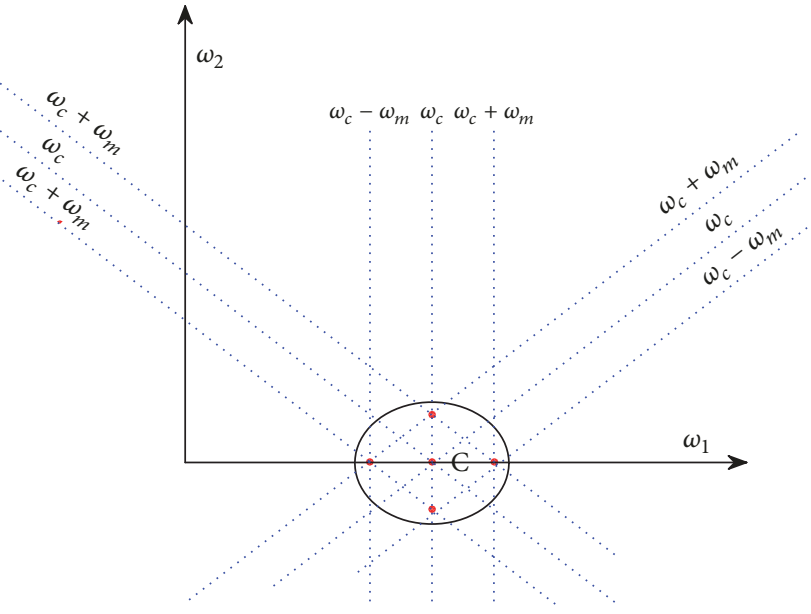

(b)

FIGURE 1: Theoretical comparison between CB (a) and MSB (b) for analyzing the modulated signal of (6).

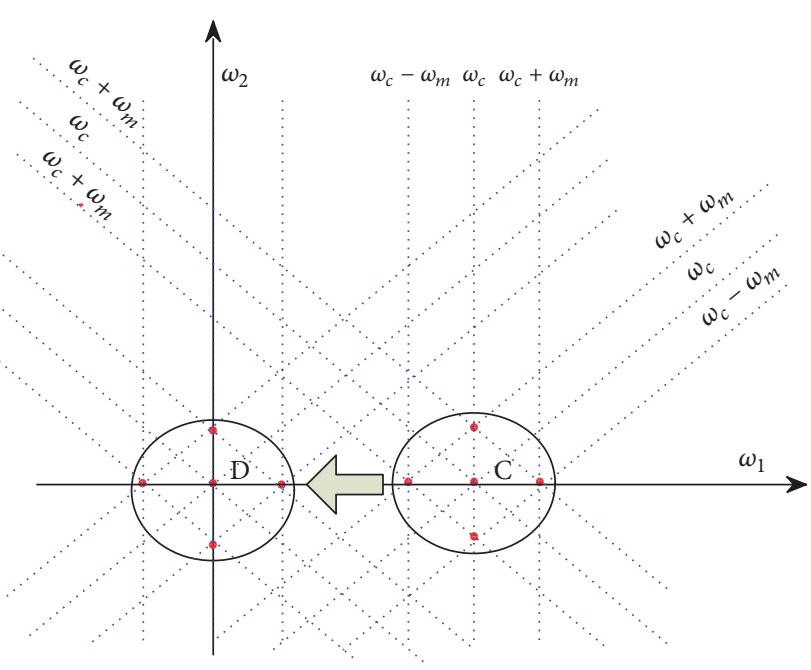

(a)

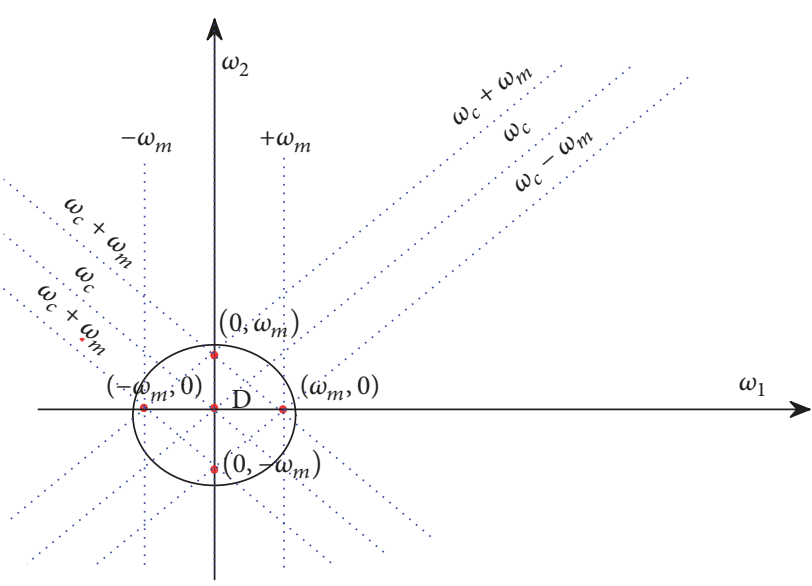

(b)

Figure 2: Theoretical shift-frequency MSB.

opposite direction; the shift frequency is shown in Figure 2, so SF-MSB can be descripted by

$$
\begin{aligned}
& B_{\mathrm{SF}-\mathrm{MS}}\left(\omega_{1}, \omega_{2}\right)=E\left\langle X\left(\omega_{1}+\omega_{2}-\omega_{c}\right)\right. \\
& \left.\cdot X\left(\omega_{1}-\omega_{2}-\omega_{c}\right) X^{*}\left(\omega_{2}-\omega_{c}\right) X^{*}\left(\omega_{2}-\omega_{c}\right)\right\rangle .
\end{aligned}
$$

The intersection points' coordinates of the SF-MSB are $\left(0,-\omega_{m}\right),(0,0),\left(0, \omega_{m}\right),\left(0, \omega_{m}\right)$, and $\left(0,-\omega_{m}\right)$; carrier frequency $\omega_{c}$ has disappeared. If the negative value points and the origin of coordinates are removed, the remaining points on the axis are the modulated frequency.

Based on the above analysis, the advantages of SF-MSB include advantages of MSB, and redundant information further decreases. Meanwhile, bispectrum only includes concerned modulation frequency; consequently when modulation frequency is recognized, frequency value on the axis is real modulation frequency, and there is no need to add or subtract carrier frequency. For complex mechanical vibration signals with multiple modulation frequencies, the analysis efficiency of demodulation identification will be greatly enhanced.

\section{Sparse Modulation Signal Bispectrum (S-MSB)}

For vibration signal of roll element bearing, fault signal has multiharmonics generally. When fault happens, multiharmonics and interference frequency exist simultaneously, which will affect the MSB and SF-MSB methods' diagnosis.

3.1. Harmonics' Effect Analysis of Modulation Signal. In (7), assuming constant $A_{0}=1, A_{1}=1$, carrier frequency $\omega_{c}=$ $50 \mathrm{~Hz}$, modulation frequency $\omega_{m 1}=8 \mathrm{~Hz}, \omega_{m 2}=15 \mathrm{~Hz}$, and 
interference frequency $\omega_{h}=11 \mathrm{~Hz}, \theta$ and $\varphi$ are zero, so the signals are simulated as

$$
\begin{aligned}
& x_{1}(t)=\left[1+\cos \left(2 \pi \omega_{m 1} t\right)\right] \cos \left(2 \pi \omega_{c} t\right)+0.9 \\
& \cdot \cos \left(2 \pi \omega_{h} t\right)+1.8 n(t) \\
& x_{2}(t)=\left[1+\cos \left(2 \pi \omega_{m 1} t\right)+0.8 \cos \left(4 \pi \omega_{m 1} t\right)\right] \\
& \cdot \cos \left(2 \pi \omega_{c} t\right)+0.9 \cos \left(2 \pi \omega_{h} t\right)+1.8 n(t) \\
& x_{3}(t)=\left[1+\cos \left(2 \pi \omega_{m 1} t\right)+0.8 \cos \left(4 \pi \omega_{m 1} t\right)\right. \\
& \left.+0.6 \cos \left(6 \pi \omega_{m 1} t\right)\right] \cos \left(2 \pi \omega_{c} t\right)+0.9 \cos \left(2 \pi \omega_{h} t\right) \\
& +1.8 n(t) \\
& x_{4}(t)=\left[1+\cos \left(2 \pi \omega_{m 1} t\right)+0.8 \cos \left(4 \pi \omega_{m 1} t\right)\right. \\
& \left.+0.6 \cos \left(6 \pi \omega_{m 1} t\right)+\cos \left(2 \pi \omega_{m 2} t\right)\right] \cos \left(2 \pi \omega_{c} t\right) \\
& +0.9 \cos \left(2 \pi \omega_{h} t\right)+1.8 n(t) \\
& x_{5}(t)=\left[1+\cos \left(2 \pi \omega_{m 1} t\right)+0.8 \cos \left(4 \pi \omega_{m 1} t\right)\right. \\
& +0.6 \cos \left(6 \pi \omega_{m 1} t\right)+\cos \left(2 \pi \omega_{m 2} t\right) \\
& \left.+0.8 \cos \left(4 \pi \omega_{m 2} t\right)\right] \cos \left(2 \pi \omega_{c} t\right)+0.9 \cos \left(2 \pi \omega_{h} t\right) \\
& +1.8 n(t) \\
& x_{6}(t)=\left[1+\cos \left(2 \pi \omega_{m 1} t\right)+0.8 \cos \left(4 \pi \omega_{m 1} t\right)\right. \\
& +0.6 \cos \left(6 \pi \omega_{m 1} t\right)+\cos \left(2 \pi \omega_{m 2} t\right) \\
& \left.+0.8 \cos \left(4 \pi \omega_{m 2} t\right)+0.6 \cos \left(6 \pi \omega_{m 2} t\right)\right] \cos \left(2 \pi \omega_{c} t\right) \\
& +0.9 \cos \left(2 \pi \omega_{h} t\right)+1.8 n(t) \text {. }
\end{aligned}
$$

Here, $x_{1}(t), x_{2}(t)$, and $x_{3}(t)$ are modulation signal containing frequency $\omega_{m 1}$ and its second harmonics and third harmonic, $x_{4}(t), x_{5}(t), x_{6}(t)$, are frequency $\omega_{m 2}$ and second harmonics and third harmonic based on $x_{3}(t), n(t)$ are random noise. After Fourier transformation and MSB transformation, the power spectrum and MSB of modulation signal $x_{1}(t) \sim x_{6}(t)$ are as shown in Figure 3(1) (6); $f_{c}$ represents the carrier frequency and $f_{x}$ represents the signal frequency.

From Figure 3, it can be seen that spectrum's distribution in $f_{c}$-axis is the same as power spectrum; the modulation frequency and its harmonics are distributed on both sides of the carrier frequency $\omega_{c}=50 \mathrm{~Hz}$, expressed as $\omega_{c}, \omega_{c} \pm$ $\omega_{m 1}, \omega_{c} \pm 2 \omega_{m 1}, \omega_{c} \pm 3 \omega_{m 1}, \omega_{c} \pm \omega_{m 2}, \omega_{c} \pm 2 \omega_{m 2}$, and $\omega_{c} \pm$ $3 \omega_{m 2}$; interference frequency $\omega_{h}=11 \mathrm{~Hz}$ is unchanged and random noise $n(t)$ is effectively eliminated. $\omega_{m 1}, \omega_{m 2}$ are two modulation frequencies.

Figure 4 expresses the horizontal projection of $x_{1}(t) \sim x_{6}(t)$ MSB. According to Figures 3 and 4 , when signal has only one modulation frequency $\omega_{m 1}$ and its harmonics, if frequency on $f_{c}$-axis is moved away, the other frequencies are mainly concentrated on $f_{c}=\omega_{c}$. When frequency $\omega_{m 2}$ and its harmonics are added, the interference frequency on $f_{c}=\omega_{h}$ increases rapidly; other unpredictable frequency components also flocked to appear.
And the signal frequency distribution on $f_{c}=\omega_{c}$ becomes also very dense, even showing frequency aliasing such as $\omega_{m 2}=15 \mathrm{~Hz}$ and $2 \omega_{m 1}=16 \mathrm{~Hz}$, and identification to multiple modulation frequency components becomes a bit difficult.

3.2. Idea of S-MSB. To the MSB of the signal $x_{1}(t) \sim x_{6}(t)$, if we continue to shift frequency $\omega_{c}$ in the opposite direction of $f_{c}$-axis using SF-MSB and extract the bispectrum of the first quadrant as the research object, Figure 5 will be obtained.

Figure 5 describes SF-MSB of signal $x_{1}(t) \sim x_{6}(t)$. It can be seen that frequency components on $f_{c}$-axis express the power spectrum of the demodulated signal, and frequency components on $f_{x}$-axis express demodulated signal's spectrum on $f_{c}=\omega_{c}$ after MSB treatment. On the diagonal, there is no frequency component to SF-MSB of signal $x_{1}(t)$ in Figure $5(1)$, but there is $\omega_{m 1}=8 \mathrm{~Hz}$ to SF-MSB of signal $x_{2}(t)$ and $x_{3}(t)$ in Figure 5(2) (3), and the harmonic frequency disappears. Similarly, on the diagonal, there is only $\omega_{m 1}=$ $8 \mathrm{~Hz}$ to SF-MSB of signal $x_{4}(t)$ in Figure 5(4); there are $\omega_{m 1}$ $=8 \mathrm{~Hz}, \omega_{m 2}=15 \mathrm{~Hz}$ to SF-MSB of signals $x_{5}(t)$ and $x_{6}(t)$ in Figure 5(5) and (6), and the harmonic frequency disappears.

In Figure 5(1), there is no frequency on the diagonal of SF-MSB because $x_{1}(t)$ has only one frequency $\omega_{m 1}=8 \mathrm{~Hz}$ and has no harmonics. Similarly, in Figure 5(4), there is only one frequency $\omega_{m 1}=8 \mathrm{~Hz}$ and frequency $\omega_{m 2}=15 \mathrm{~Hz}$ does not appear on the diagonal of SF-MSB, because $x_{4}(t)$ has only harmonics of frequency $\omega_{m 1}=8 \mathrm{~Hz}$. On the other hand, no matter how many harmonics, only the fundamental frequency exists on the diagonal of SF-MSB of signals $x_{2}(t)$, $x_{3}(t), x_{5}(t)$, and $x_{6}(t)$ in Figure 5(2) (3) (5) (6). Because almost all of the vibration signals have harmonics, frequency on the diagonal of SF-MSB can be used to characterize the fundamental frequency of the modulation frequency.

For the actual vibration signal, the carrier frequency is generally the natural frequency of the equipment, which is very difficult to get accurately because of the complexity of the system. From Figures 3 and 4, it can be seen that the useful frequency is modulated to near the high-frequency carrier frequency and here is the maximum energy, but as the frequency component increases, the interference frequency in the low-frequency part is constantly present and enhanced. So low-frequency interference is removed by frequency domain filter in MSB, and then the entire bispectrum is summed to the $f_{c}$-axis, lastly taking frequency at the maximum sum as carrier frequency.

This method enhances the fundamental frequency and reduces the harmonic frequency, which makes much less frequency components and the distribution becomes sparse on the diagonal of SF-MSB on the vibration signal with complicated frequency components in the actual mechanical system. Based on this, we call this method sparse modulation signal bispectrum (S-MSB) analysis. The steps are as follows:

(1) Obtain the MS-bispectrum by MSB analysis.

(2) Compute carrier frequency by summing to the $f_{c}$-axis on bispectrum after frequency domain filter.

(3) Shift frequency on MSB of signal. 




— Noise-free

Added Noise

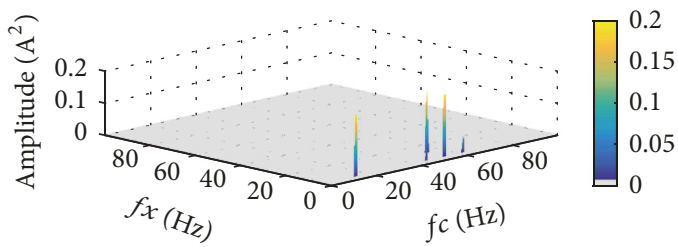

(1) Power spectrum and MSB of $x_{1}(t)$

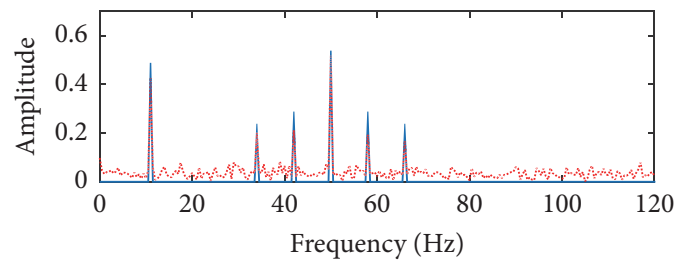

— Noise-free Added Noise

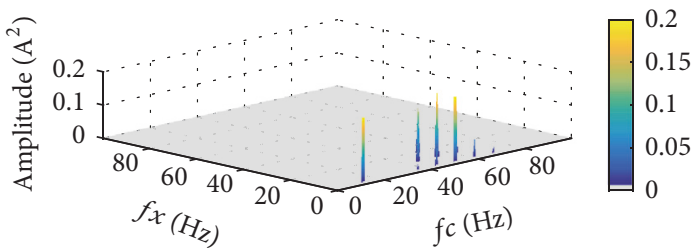

(2) Power spectrum and MSB of $x_{2}(t)$

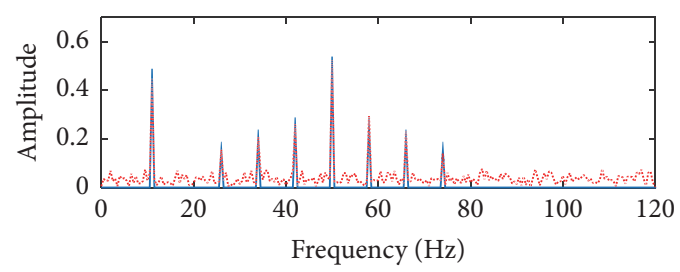

_ Noise-free

..... Added Noise

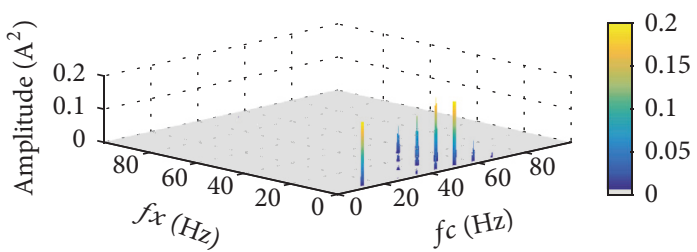

(3) Power spectrum and MSB of $x_{3}(t)$

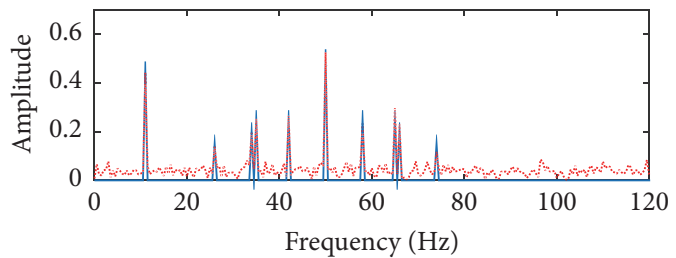

— Noise-free

Added Noise

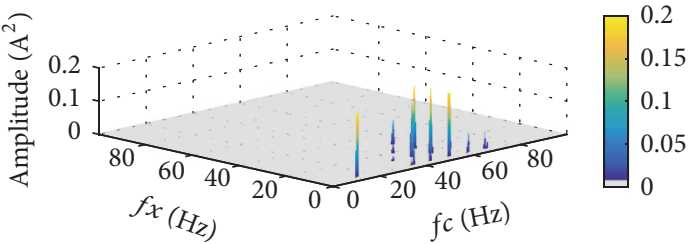

(4) Power spectrum and MSB of $x_{4}(t)$

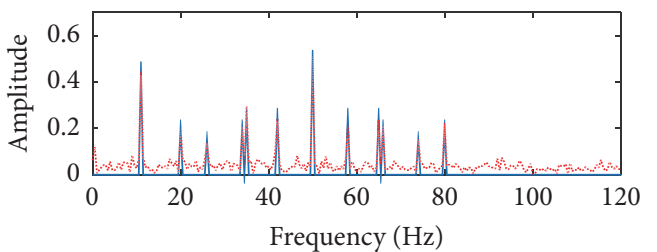

- Noise-free

Added Noise

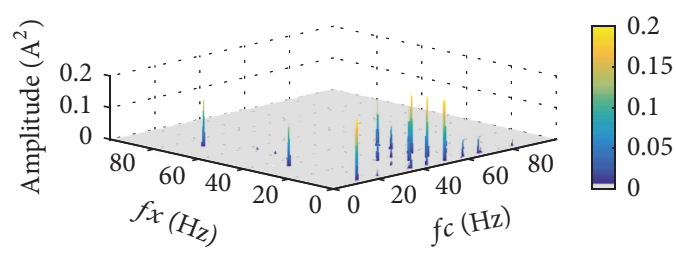

(5) Power spectrum and MSB of $x_{5}(t)$

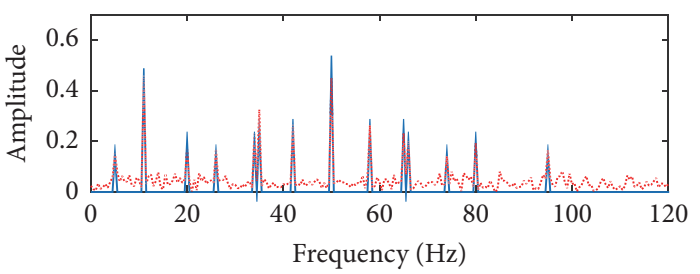

- Noise-free

Added Noise



(6) Power spectrum and MSB of $x_{6}(t)$

Figure 3: Power spectrum and MSB of signal $x_{1}(t) \sim x_{6}(t)$. 


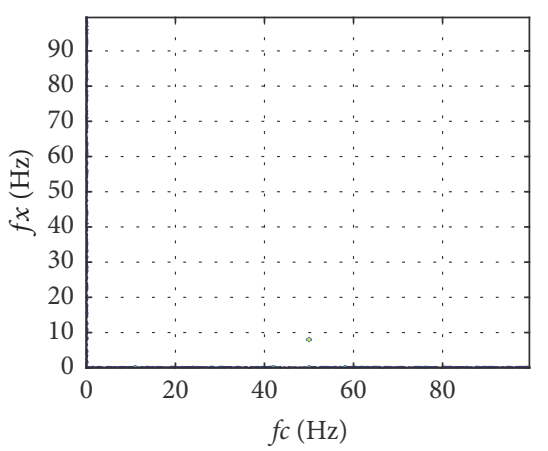

(1)

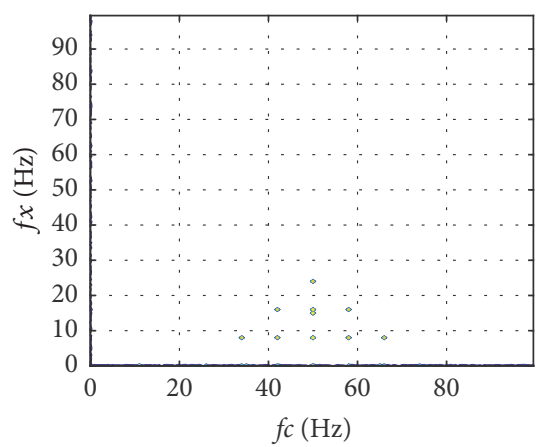

(4)

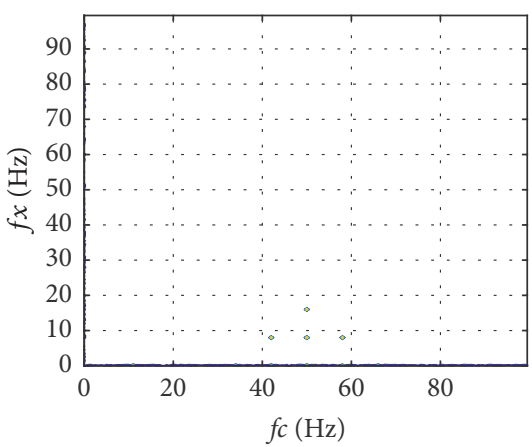

(2)



(5)

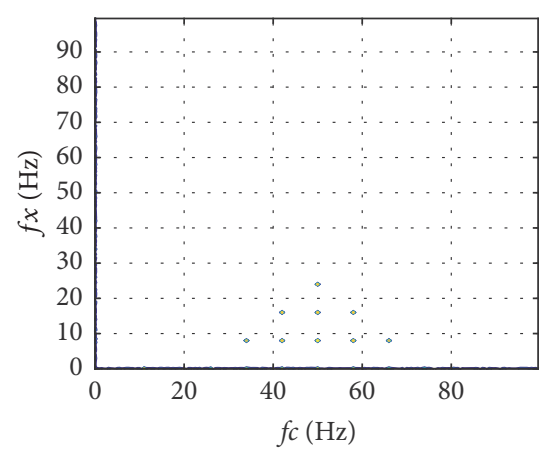

(3)



(6)

FIgURE 4: Horizontal projection of MSB of signal $x_{1}(t) \sim x_{6}(t)(1 \sim 6)$.

(4) Obtain the useful modulation frequency by the means of diagonal slices on SF-MSB.

3.3. Demodulation Analysis of S-MSB on the Simulation Signal. According to the steps of S-MSB, the slice spectrum of the simulation signal $x_{1}(t) \sim x_{6}(t)$ is shown in Figure $6(1) \sim(6)$. In each graph, the top is the diagonal slice which represents the fundamental frequency of the modulated signal, the middle is the horizontal slice which represents the power spectrum after moving frequency, and the bottom is the vertical slice which represents the power spectrum after MSB demodulation.

In Figure 6(1), because $x_{1}(t)$ has no harmonics, there is no frequency on the slice spectrum of S-MSB, but $\omega_{m 1}=8$ exists on the power spectrum slice after moving frequency and MSB demodulation, and the latter is more obvious. In Figure 6(2) and (3), to signal $x_{2}(t), x_{3}(t), \omega_{m 1}=8$ appears and does not increase as the number of harmonics increases the slice spectrum of S-MSB, but $\omega_{m 1}=8,2 \omega_{m 1}=16$, and $3 \omega_{m 1}=$ 24 exist on the power spectrum slice after moving frequency and MSB demodulation. Similarly, in Figure 6(4) (6), when $\omega_{m 2}=15$ is added, the regular pattern of slice spectrum is the same as in Figure 6(1) (3). Therefore, by comparing the bispectrum slices of S-MSB and MSB, the modulation frequency can be identified more effectively and is immune to noise, interference, and frequency aliasing of $\omega_{m 2}=15$ and $2 \omega_{m 1}=16$.

\section{Single Fault Diagnosis Experiment of Rolling Element Bearing}

In this section, single fault data are used to verify the previous theoretical analysis and simulation. Sparse MSB analysis is proved to be effective in qualitative fault diagnosis of rolling element bearings. Fault data come from bearing data center site of the Case Western Reserve University. Bearing type and related parameters are as shown in Table 1.

In Figure 7 , the fault frequency is modulated to near $3000 \mathrm{~Hz}$ and frequency band is approximately $800 \mathrm{~Hz}$. According to the carrier frequency calculation method proposed in this paper, the inner race fault carrier frequency is taken as $2944 \mathrm{~Hz}$ and the outer race fault carrier frequency is taken as $3455 \mathrm{~Hz}$. MSB and SF-MSB are shown in Figures 8(a) and 9(a); the S-MSB spectrum, demodulated power spectrum, and MSB slice spectrum are as shown as the top, middle, and bottom in Figures 8 (b) and 9(b). On the SMSB spectrum, fault frequencies $162 \mathrm{~Hz}$ and $108 \mathrm{~Hz}$ are very obvious; harmonics do not interfere. However on demodulated power spectrum and MSB slice spectrum, not only do the fault frequency harmonics appear, but also the harmonics of the low-frequency interference appear; this will affect diagnostic effect when frequency components of signal become more complex. The experiments show that S-MSB has the better diagnostic effect than other methods for a single fault signal of bearing. 


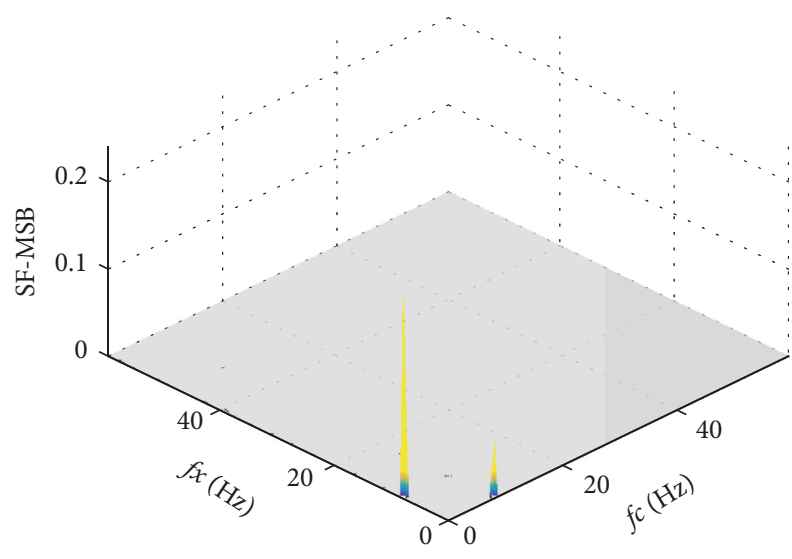

(1)

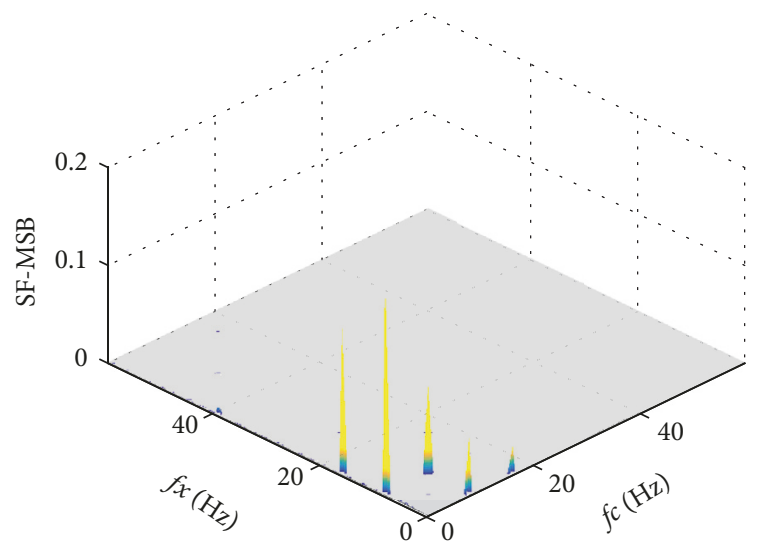

(2)

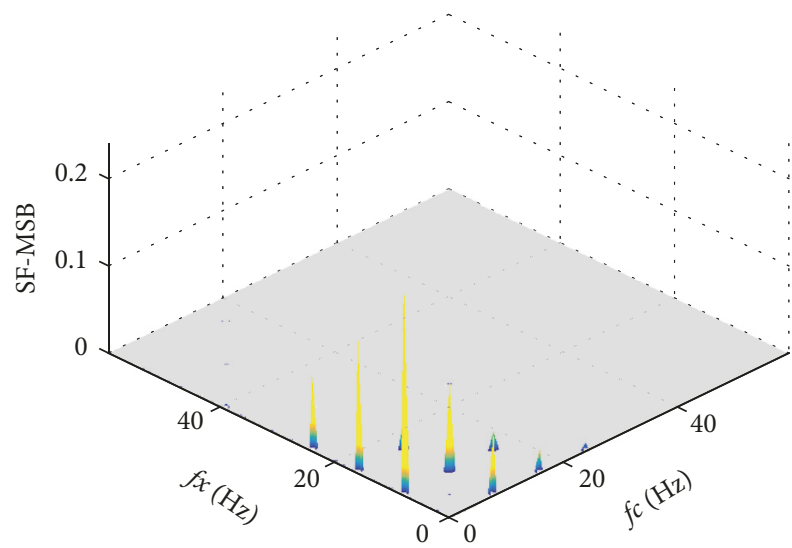

(3)

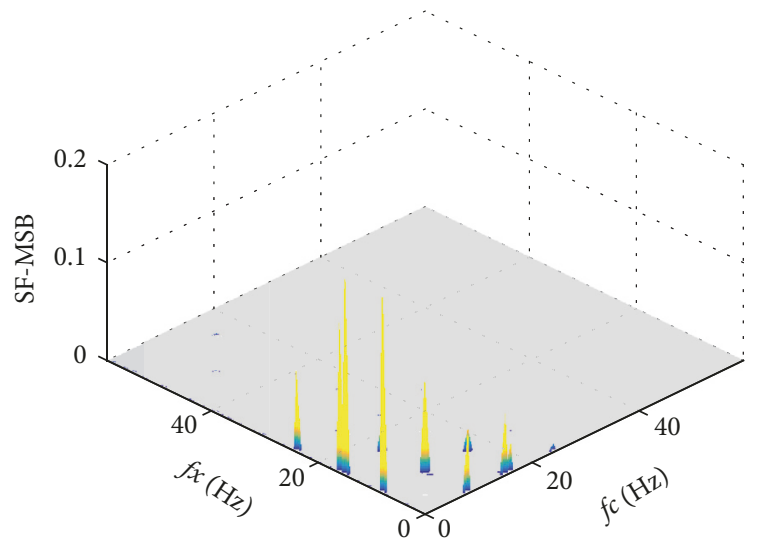

(4)



(5)



(6)

FIGURE 5: SF-MSB of signal $x_{1}(t) \sim x_{6}(t)(1 \sim 6)$.

\section{Multiple Fault Diagnosis Experiment of Rolling Element Bearing}

In order to test the recognition effect of the S-MSB method for complex fault signals, three faults on the two bearings will be identified in this section. Figure 10 is the experimental device; the right is the drive motor, drive-end bearing type is 6206 with outer race fault, and another bearing located in the middle of the left load motor and bearing seat is N406 with outer race fault and inner race fault. Rolling bearing parameters are as shown in Table 2.

In Figure 11(a), the fault frequency is modulated to near $4000 \mathrm{~Hz}$ and frequency band is approximately $1000 \mathrm{~Hz}$. After calculation, carrier frequency is taken as $4703 \mathrm{~Hz}$; MSB of 


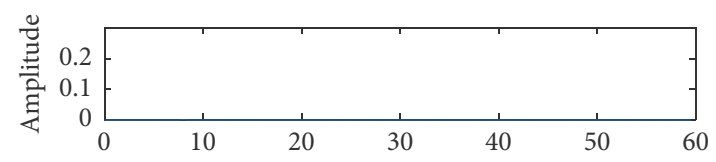

Sparse Bispectrum slice of demodulation signal $f_{\mathcal{c}}(\mathrm{Hz})$


(1)

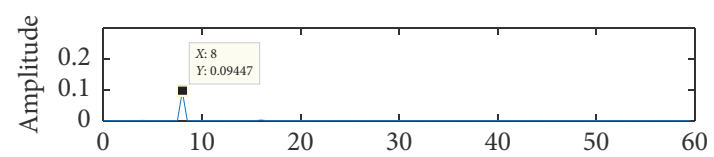

Sparse Bispectrum slice of demodulation signal $f_{\mathcal{c}}(\mathrm{Hz})$
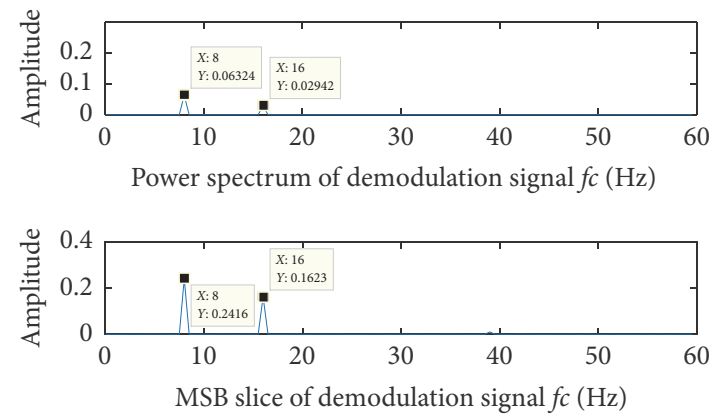

(2)


(3)
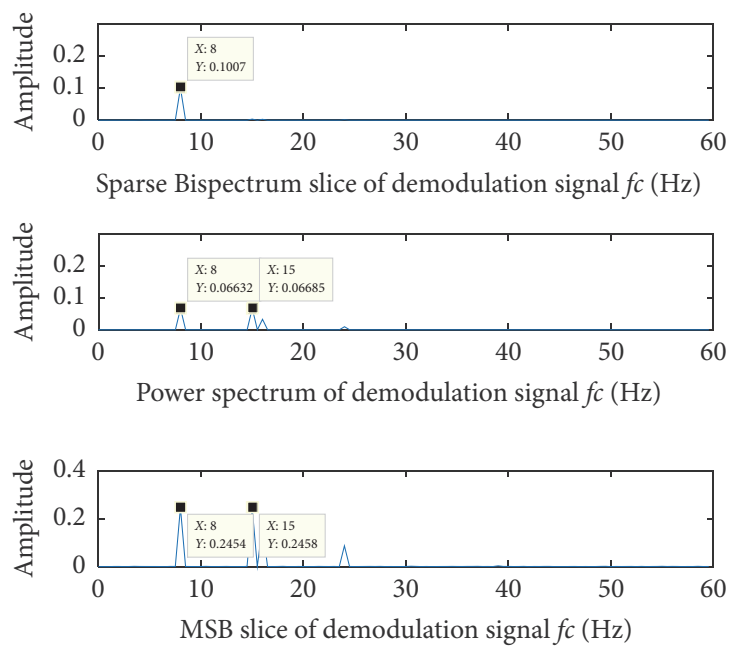

(4)

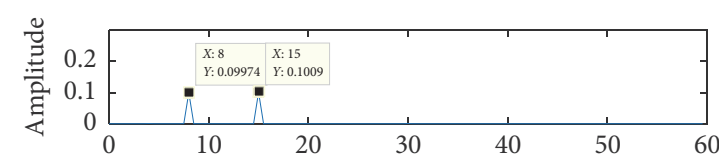

Sparse Bispectrum slice of demodulation signal $f_{\mathcal{c}}(\mathrm{Hz})$
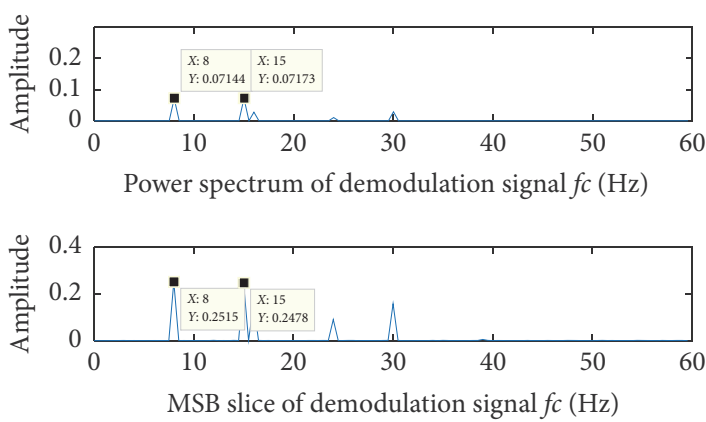

(5)
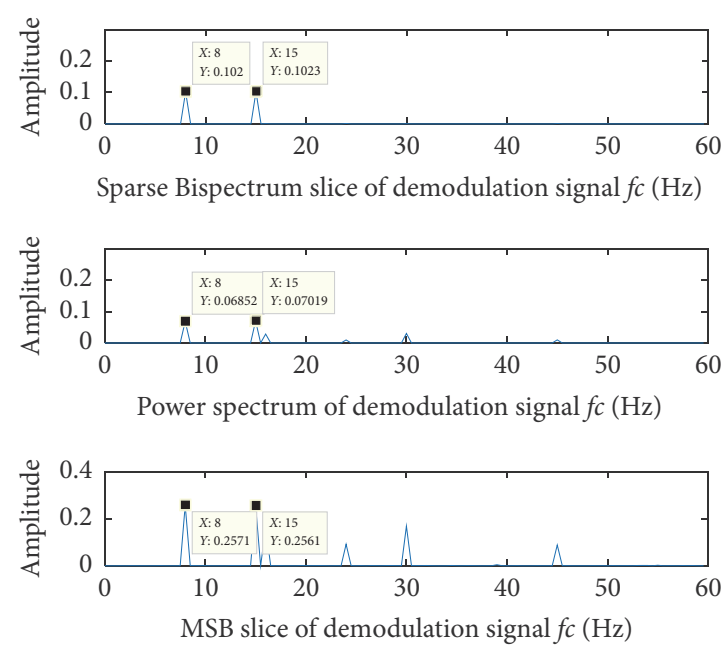

(6)

FIgURE 6: S-MSB slices of signal $x_{1}(t) \sim x_{6}(t)(1 \sim 6)$. 
TABLE 1: Motor rolling element parameters.

\begin{tabular}{|c|c|c|c|c|c|c|}
\hline Bearing type & $\begin{array}{l}\text { Rotating speed } \\
\quad(\mathrm{rpm})\end{array}$ & $\begin{array}{l}\text { Motor load } \\
\quad(\mathrm{HP})\end{array}$ & $\begin{array}{l}\text { Fault size } \\
\text { (inch) }\end{array}$ & $\begin{array}{c}\text { Sampling } \\
\text { frequency } \\
(\mathrm{Hz})\end{array}$ & $\begin{array}{l}\text { Inner race fault } \\
\text { frequency }(\mathrm{Hz})\end{array}$ & $\begin{array}{l}\text { Outer race fault } \\
\text { frequency }(\mathrm{Hz})\end{array}$ \\
\hline 6205-2RS & 1797 & 1 & $0.007^{\prime \prime}$ & 12000 & 162.1852 & 107.3648 \\
\hline
\end{tabular}

TABLE 2: Rolling element parameters.

\begin{tabular}{lccccc}
\hline Bearing type & $\begin{array}{c}\text { Rotating speed } \\
(\mathrm{rpm})\end{array}$ & $\begin{array}{c}\text { Fault size } \\
(\mathrm{mm})\end{array}$ & $\begin{array}{c}\text { Sampling } \\
\text { frequency }(\mathrm{Hz})\end{array}$ & $\begin{array}{c}\text { Outer race fault } \\
\text { frequency }(\mathrm{Hz})\end{array}$ & $\begin{array}{c}\text { inner race fault } \\
\text { frequency }(\mathrm{Hz})\end{array}$ \\
\hline N406 & 1460 & 0.18 & 96000 & 139 & 86 \\
6206 & 1460 & 0.2 & 96000 & 89 & - \\
\hline
\end{tabular}
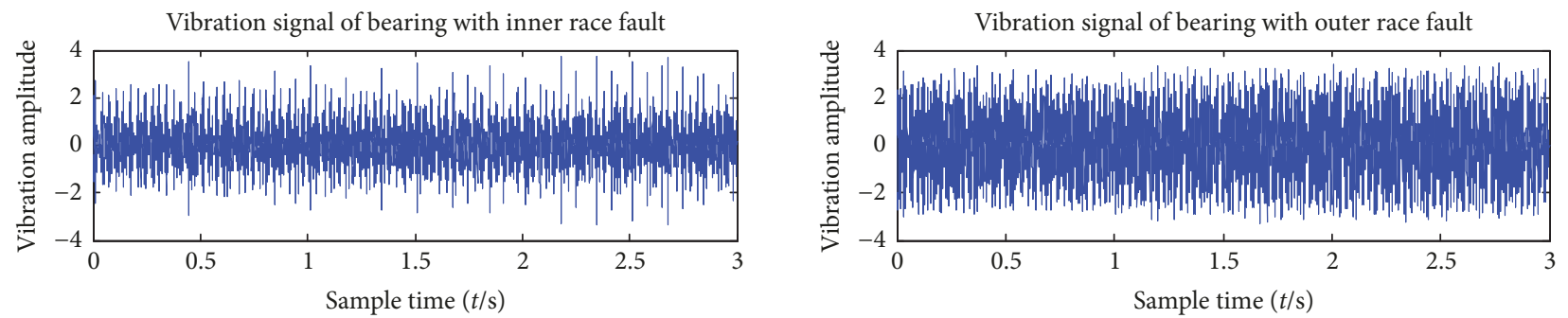

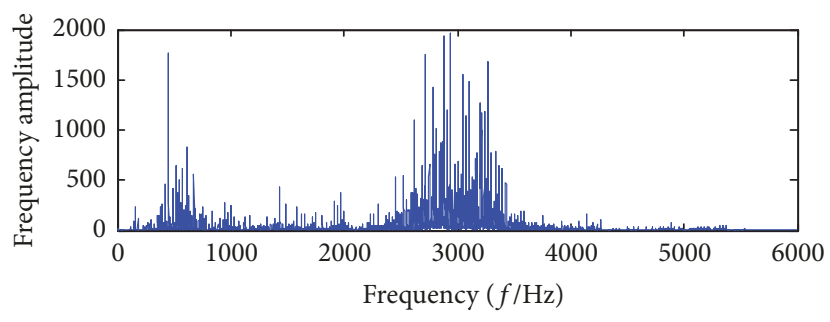

(a)

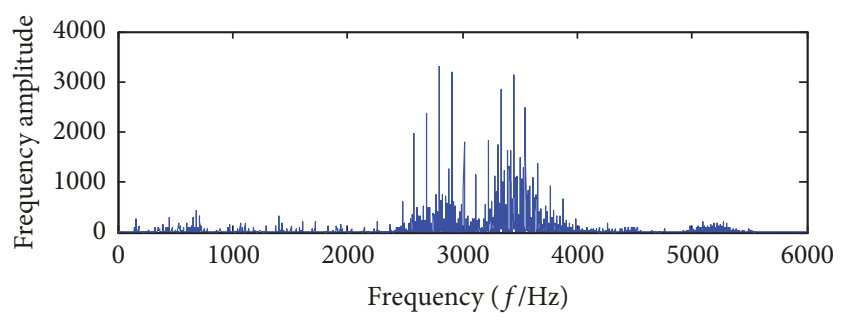

(b)

FIGURE 7: Time-domain waveform: spectrum of the inner race fault signal (a) and outer race fault signal (b).


(a)
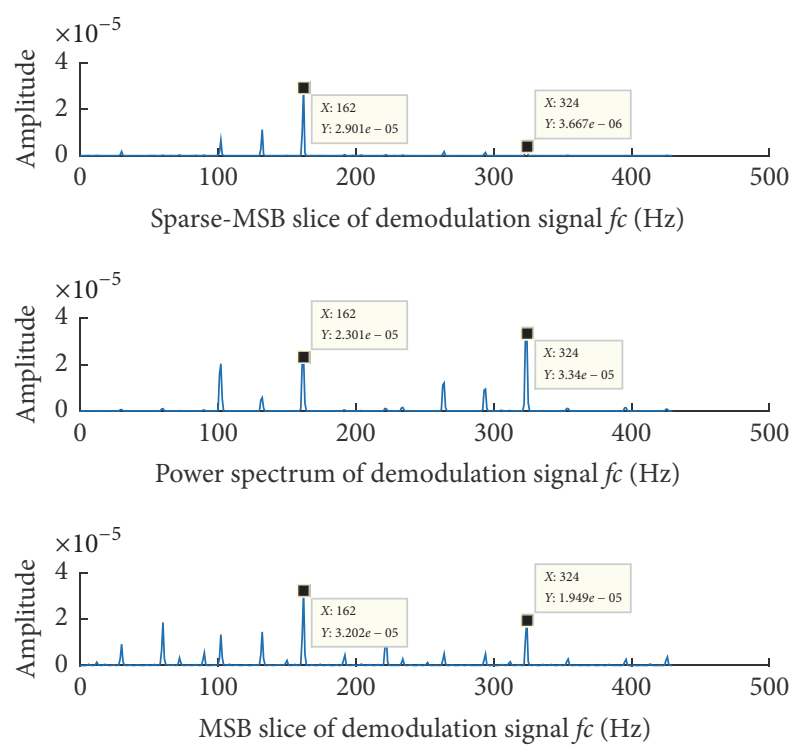

(b)

Figure 8: MSB, SF-MSB (a), and S-MSB slice, power spectrum, and MSB slice (b) of the inner race fault signal. 



(a)
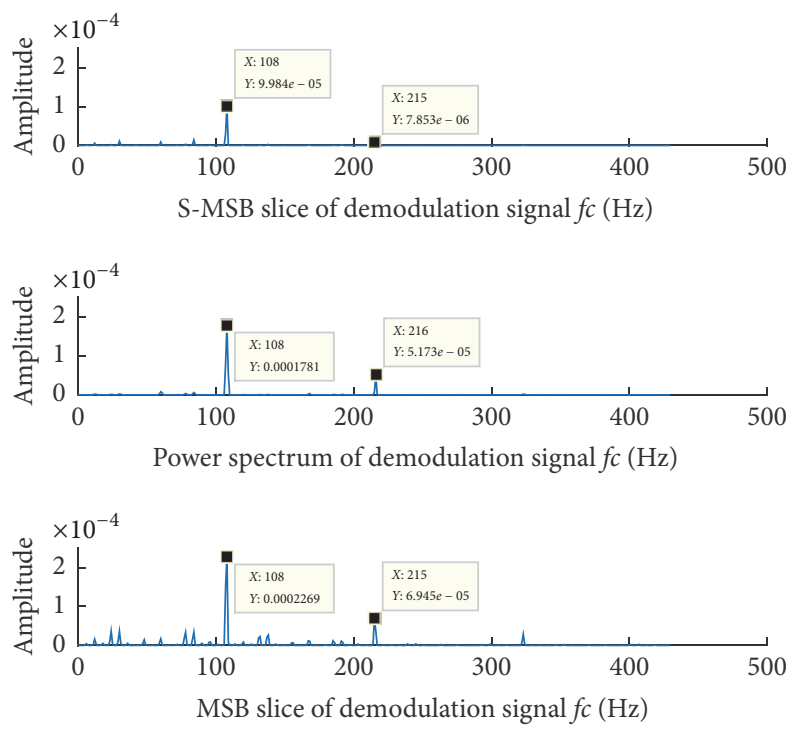

(b)

Figure 9: MSB, SF-MSB (a), and S-MSB slice, power spectrum, and MSB slice (b) of the inner race fault signal.

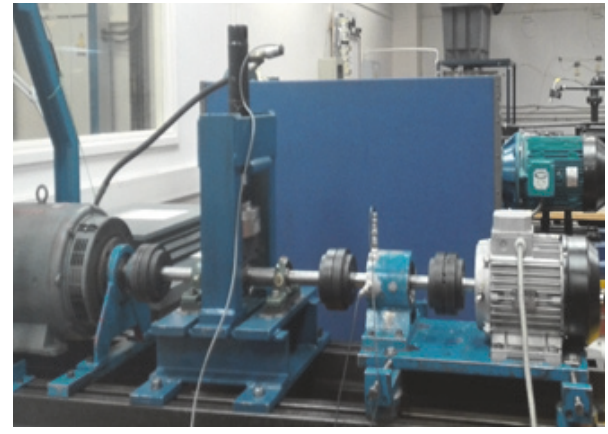

FIGURE 10: Experimental device.

this complex fault signal is as shown in Figure 11(b). By shift frequency, the SF-MSB is obtained and shows obvious symmetrical characteristics centered on the diagonal line.

The S-MSB spectrum on the upper graph of Figure 12(b) can be obtained by the means of the diagonal slices of SFMSB; the demodulated signal's power spectrum on the middle graph of and the MSB slice spectrum on the bottom graph of Figure 12(b) can be obtained by taking the horizontal and vertical axes. From Figure 12(b), it can be seen that there are a lot of trouble to complex multifault identification; each of the three subgraphs has a variety of frequency components. For S-MSB spectrum in the upper graph, three characteristic frequencies $86 \mathrm{~Hz}, 89 \mathrm{~Hz}$, and $139 \mathrm{~Hz}$ can be accurately and clearly displayed, but the demodulated power spectrum in the middle graph and the MSB slice spectrum in the low graph, although being $86 \mathrm{~Hz}$ and $89 \mathrm{~Hz}$, can be displayed; however $139 \mathrm{~Hz}$ can not only be correctly obtained (one is $138 \mathrm{~Hz}$ and the other is $137 \mathrm{~Hz}$ ), but also be distorted to the demodulated power spectrum. Therefore, the S-MSB method has the best fault identification effect.

\section{Conclusion}

In this paper, the sparse MSB is studied for its applications in fault diagnosis of rolling element bearings. The vibration signals of rolling element bearings with faults have modulation characteristics; the carrier frequencies are generally determined by inherent structure of bearing. When the carrier frequency is calculated and determined, S-MSB is more suitable for analyzing complex modulated signals than the MSB method.

Firstly, the vibration signal is demodulated by MSB analysis and its bispectrum is obtained. By MSB analysis, wideband noise in bearing vibration signals is suppressed effectively so that the fault frequency can be obtained more accurately. Secondly, SF-MSB is obtained by shifting the carrier frequency, which reduces redundant information further; the fault frequency is the actual value on the coordinate and there is no need to add or subtract carrier frequency; the analysis efficiency is greatly enhanced. At last, diagonal slices were performed to obtain a sparse representation of MSB, which can effectively eliminate the harmonic frequency and improve the efficiency and effect of fault frequency identification of complex modulation signals.

S-MSB is an improved method based on the MSB, which proposed the SF-MSB, the carrier frequency calculation, and the diagonal slice sparse demodulation method. In the bearing single fault and multifault feature frequency extraction, S-MSB method shows the better results.

\section{Conflicts of Interest}

The authors declare that they have no conflicts of interest. 




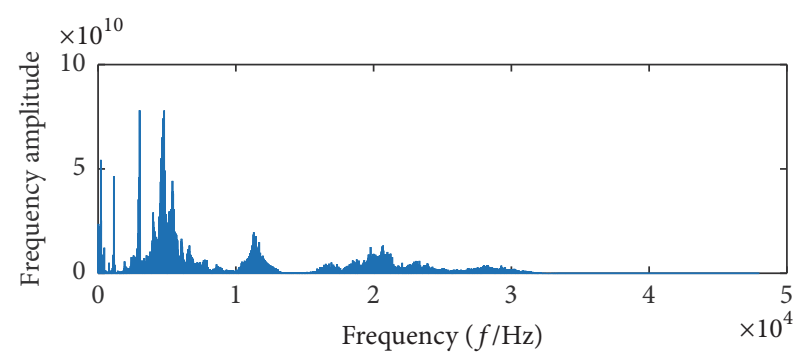

(a)

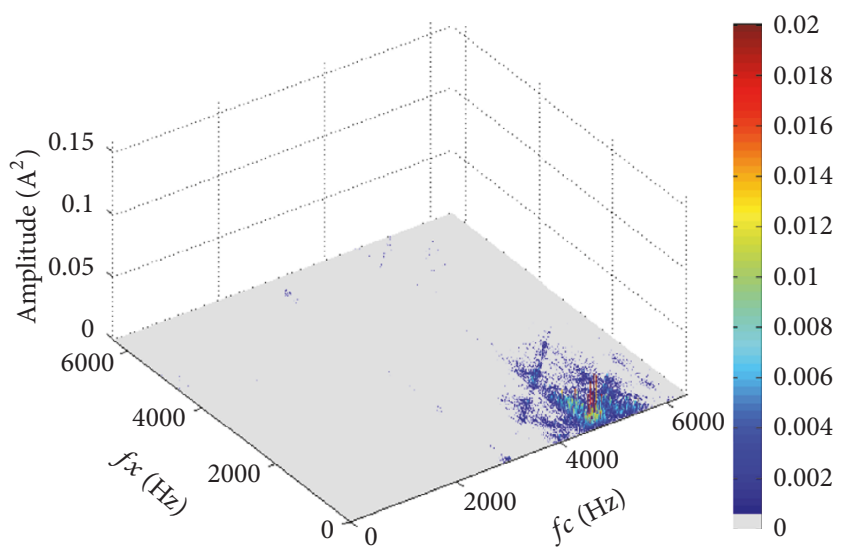

(b)

Figure 11: Time-domain waveform, spectrum (a) and MSB (b) of the complex fault signal.

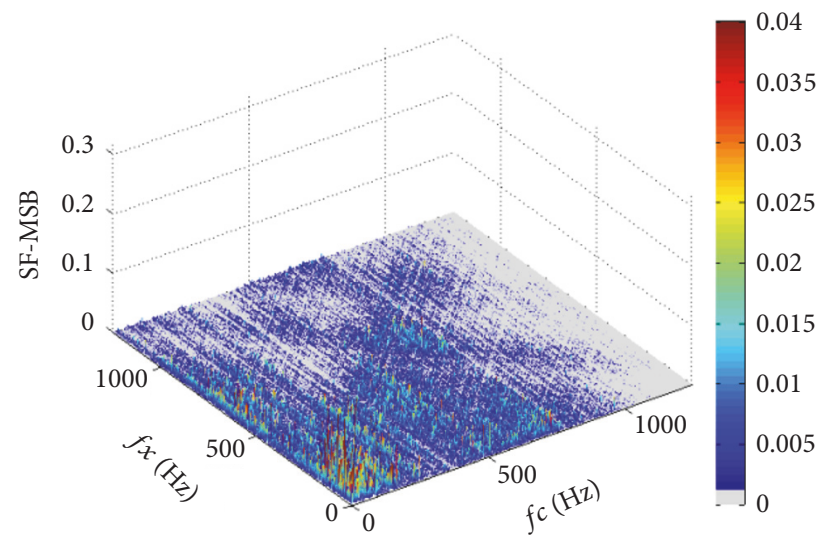

(a)
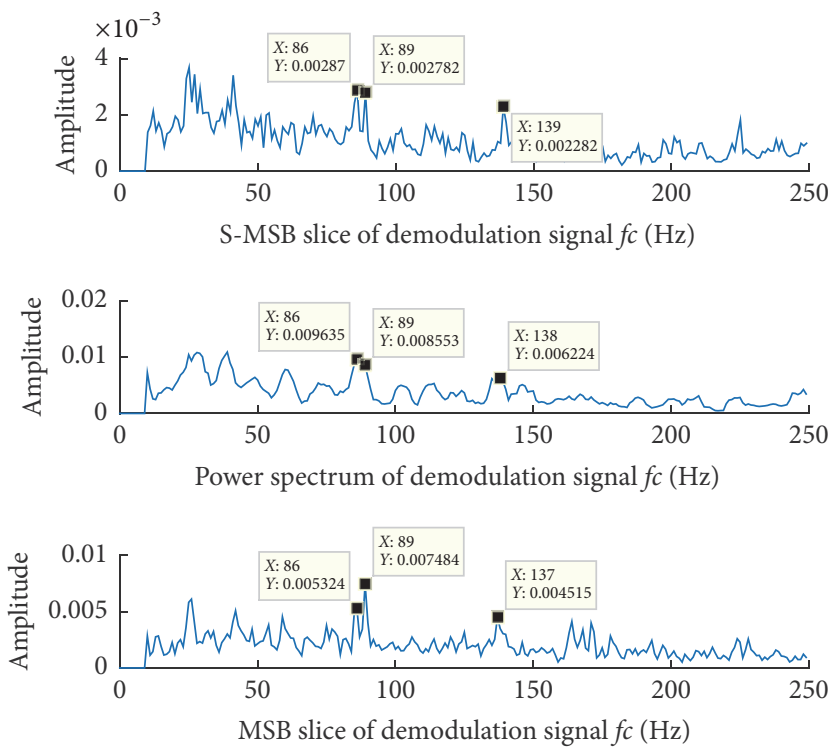

(b)

FIGURE 12: (a) SF-MSB of demodulation signal to the complex fault. (b) S-MSB spectrum slice, power spectrum slice; MSB slice of demodulation signal to the complex fault.

\section{Acknowledgments}

Financial support from National Natural Science Foundation of China $(51575178,51175170)$ and financial support from China Scholarship Fund (201408430118) are appreciated.

\section{References}

[1] Y. Jiangtian, C. Jiaji, and Z. Ziping, "Bispectral analysis and its application in machinery diagnosis," China Mechanical Engineering, vol. 11, no. 4, pp. 424-426, 2000.

[2] J. Li, J. Han, Z. Li, and W. Hao, "Bispectrum analysis in the wavelet transform domain and its application to the fault diagnosis of rolling bearings," Journal of Vibration and Shock, vol. 25, no. 5, pp. 92-95, 2006.

[3] R. Piotrkowski, E. Castro, and A. Gallego, "Wavelet power, entropy and bispectrum applied to $\mathrm{AE}$ signals for damage identification and evaluation of corroded galvanized steel," Mechanical Systems and Signal Processing, vol. 23, no. 2, pp. 432445, 2009.

[4] S. E. El-Khamy and H. A. Elsayed, "Classification of MultiUser Chirp Modulation Signals Using Wavelet Higher-OrderStatistics Features and Artificial Intelligence Techniques," International Journal of Communications, Network and System Sciences, vol. 05, no. 09, pp. 520-533, 2012. 
[5] Z. Liu, D. Yu, and J. Liu, "Order bispectrum analysis based on fault characteristic frequency and its application to the fault diagnosis of rolling bearings," Zhongguo Dianji Gongcheng Xuebao/Proceedings of the Chinese Society of Electrical Engineering, vol. 11, no. 33, pp. 123-129, 2013.

[6] L. Li, J. Han, P. Li, W. Hao, and L. Chen, "Vector-bispectrum analysis and its application in machinery fault diagnosis," Jixie Gongcheng Xuebao/Journal of Mechanical Engineering, vol. 47, no. 17, pp. 50-54, 2011.

[7] C. T. Yiakopoulos and I. A. Antoniadis, "Cyclic bispectrum patterns of defective rolling element bearing vibration response," Forschung im Ingenieurwesen, Engineering Research, vol. 70, no. 2, pp. 90-104, 2006.

[8] Y. Zhou, J. Chen, G. M. Dong, W. B. Xiao, and Z. Y. Wang, "Application of the horizontal slice of cyclic bispectrum in rolling element bearings diagnosis," Mechanical Systems and Signal Processing, vol. 26, no. 1, pp. 229-243, 2012.

[9] A. Yunusa-Kaltungo and J. K. Sinha, "Combined bispectrum and trispectrum for faults diagnosis in rotating machines," Proceedings of the Institution of Mechanical Engineers, Part O: Journal of Risk and Reliability, vol. 228, no. 4, pp. 419-428, 2014.

[10] M. Hassan, J. Tarbutton, A. Bayoumi, and Y.-J. Shin, "Condition monitoring of helicopter drive shafts using quadraticnonlinearity metric based on cross-bispectrum," IEEE Transactions on Aerospace and Electronic Systems, vol. 50, no. 4, pp. 2819-2829, 2014.

[11] G. Dong, J. Chen, and F. Zhao, "A frequency-shifted bispectrum for rolling element bearing diagnosis," Journal of Sound and Vibration, vol. 339, pp. 396-418, 2015.

[12] J. R. Stack, R. G. Harley, and T. G. Habetler, "An amplitude modulation detector for fault diagnosis in rolling element bearings," IEEE Transactions on Industrial Electronics, vol. 51, no. 5, pp. 1097-1102, 2004.

[13] F. Gu, Y. Shao, N. Hu, A. Naid, and A. D. Ball, "Electrical motor current signal analysis using a modified bispectrum for fault diagnosis of downstream mechanical equipment," Mechanical Systems and Signal Processing, vol. 25, no. 1, pp. 360-372, 2011.

[14] M. Haram, T. Wang, F. Gu, and A. D. Ball, "Electrical motor current signal analysis using a modulation signal bispectrum for the fault diagnosis of a gearbox downstream," Journal of Physics: Conference Series, vol. 364, no. 1, Article ID 012050, pp. 17426596, 2012.

[15] Z. Chen, T. Wang, F. Gu, M. Haram, and A. Ball, "Gear transmission fault diagnosis based on the bispectrum analysis of induction motor current signatures," Jixie Gongcheng Xuebao/Journal of Mechanical Engineering, vol. 48, no. 21, pp. 8490, 2012.

[16] A. Alwodai, T. Wang, Z. Chen, F. Gu, R. Cattley, and A. Ball, "A Study of Motor Bearing Fault Diagnosis using Modulation Signal Bispectrum Analysis of Motor Current Signals," Journal of Signal and Information Processing, vol. 04, no. 03, pp. 72-79, 2013.

[17] F. Gu, T. Wang, A. Alwodai, X. Tian, Y. Shao, and A. D. Ball, "A new method of accurate broken rotor bar diagnosis based on modulation signal bispectrum analysis of motor current signals," Mechanical Systems and Signal Processing, vol. 50-51, no. 1, pp. 400-413, 2015.

[18] I. Rehab, X. Tiana, N. Hu et al., "A study of two bispectral features from envelope signals for bearing fault diagnosis," in Proceedings of the 30th Manchester Conference Centre, IncoME, Manchester, 2016. 




Advances in

Operations Research

\section{-n-m}
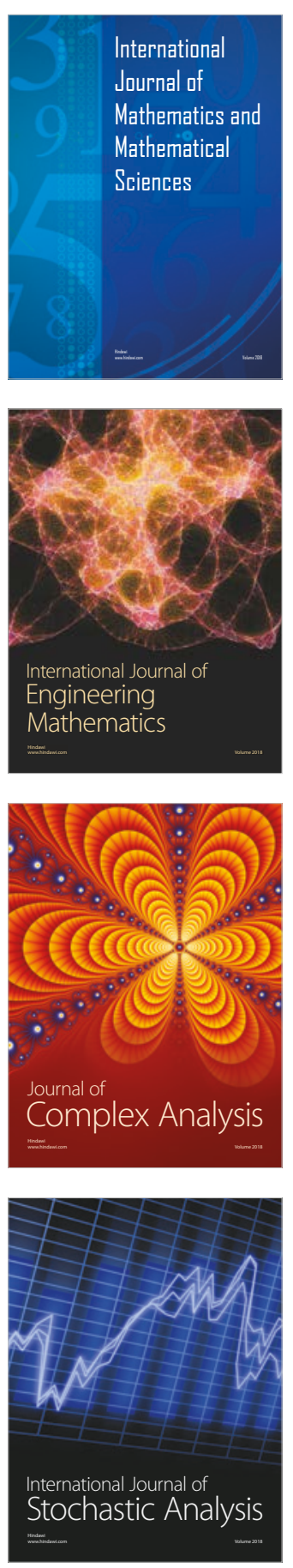
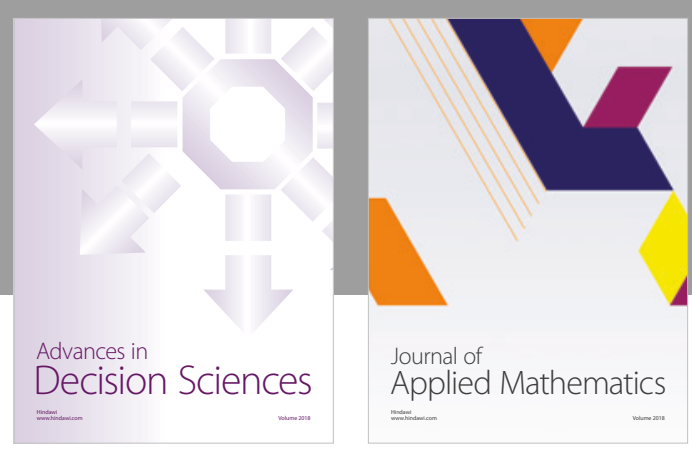

Journal of

Applied Mathematics
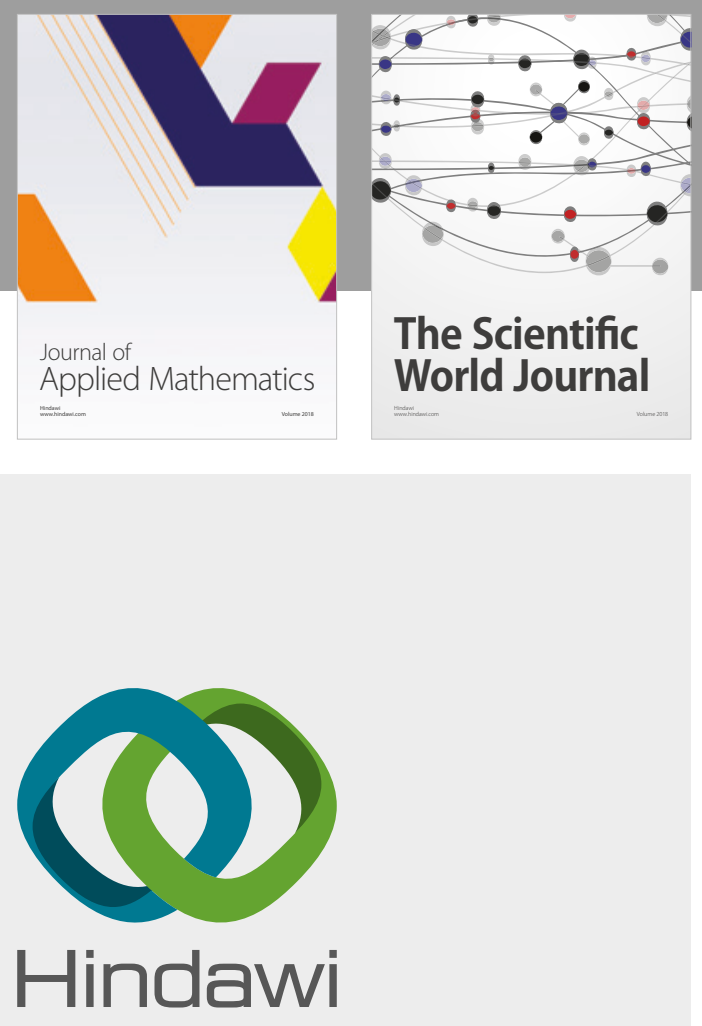

Submit your manuscripts at

www.hindawi.com

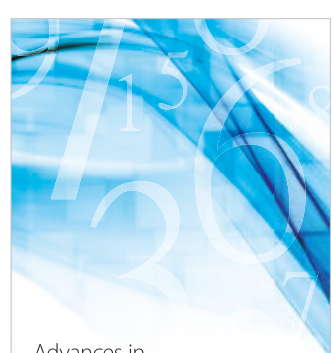

Advances in
Numerical Analysis
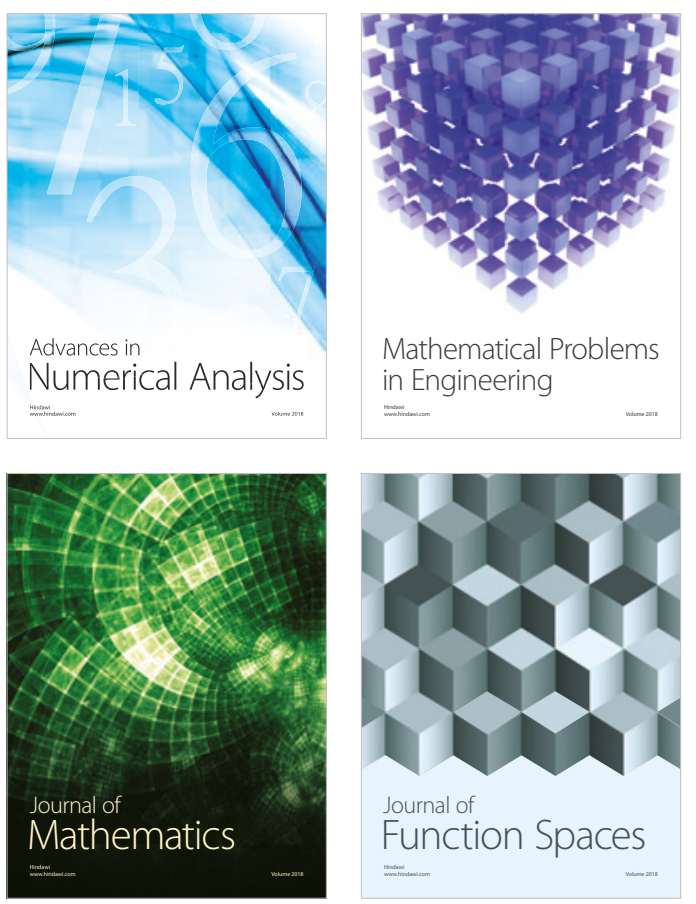

Mathematical Problems in Engineering

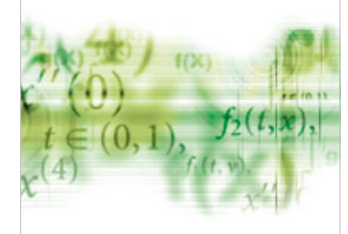

International Journal of

Differential Equations

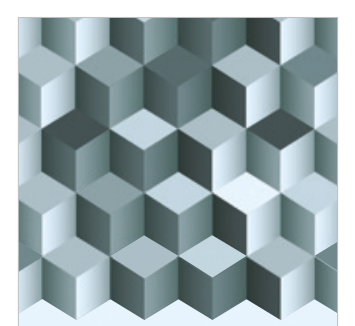

Journal of

Function Spaces

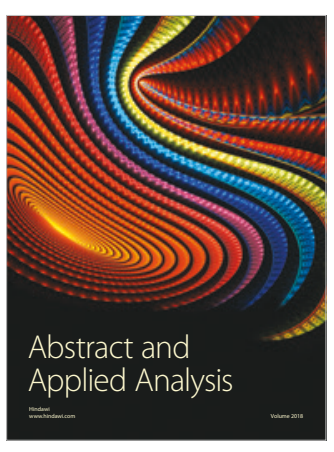

The Scientific

World Journal



Journal of

Probability and Statistics
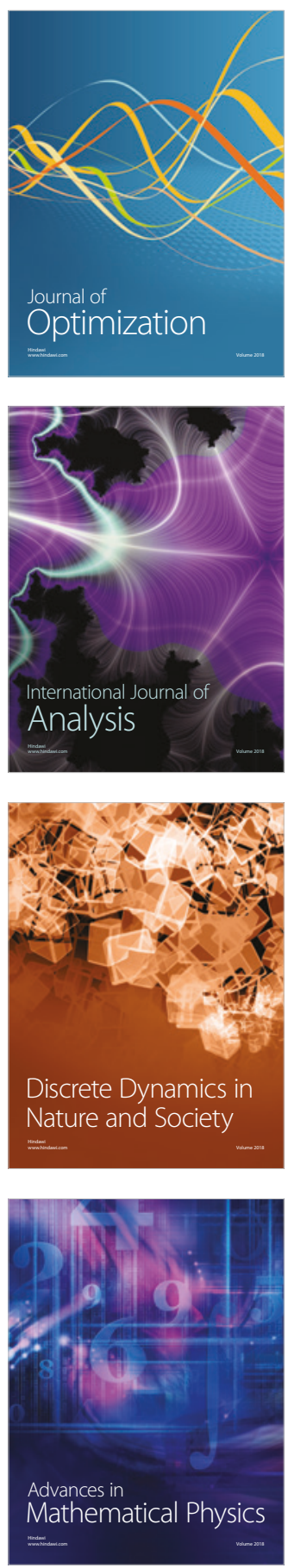